\title{
Conceptual Model of Activity as Tool for Developing a Dementia Care Support System
}

\author{
Helena Lindgren \\ Department of Computing Science, Umeå University, Sweden, helena@cs.umu.se
}

\begin{abstract}
The clinical domain of cognitive diseases and dementia is recognized by its highly complex knowledge domain, requiring expertise and experience in handling situations with a variety of symptoms and diseases, distributed over different levels in organizations and different professions. In this paper a case study is presented where the process of investigating suspected dementia in patient cases were analyzed. An early prototype of the decision-support system DMSS (Dementia Management and Support System) was integrated in the process. The main aim for the case study was to capture and model the complex target activity for the purpose of knowledge acquisition and formalization in the development of a decision-support system for the domain. The resulting model is general, in that it captures structures and required knowledge at different levels of care, however, specific enough to provide a perception of use context and semi-formal base for further development of the system for different use environments, with different local solutions to work division, etc. The results are fed into the development of DMSS and the general activity analysis framework is being developed.
\end{abstract}

Keywords: Activity theory, Work analysis, Knowledge-based systems, Decision support, Knowledge management, Dementia care

\section{Introduction}

The clinical decision-support system (CDSS) DMSS (Dementia Management Support System) is being developed for assisting medical personnel in the investigation of suspected cases of dementia [1]. The application domain is used to investigate a range of aspects concerning knowledge acquisition, knowledge formalization and representation, interaction design and methods for transforming informal clinical practice knowledge into usable support in a CDSS. The main purpose of the system is to function as an extension of the individual actor's cognitive ability and as a common ground for collaborative and distributed team 
work. The system is designed to support higher-level cognitive functions as reasoning, decision-making and learning.

The clinical domain of cognitive diseases and dementia is recognized by its highly complex knowledge domain, requiring expertise and experience in handling the progressivity of the disease, and consequently, re-occurring situations with a variety of social, cognitive, psychological and behavioral complications [1]. In an organizational perspective, work can be done differently depending on the available resources, treatment protocols and priorities. In addition, the organization of work is also subject to evolvement and change. When focus is put on the clinical activity, issues such as the distinction between facts, judgments, qualities of evidence, what is normal vs. abnormal, levels of dysfunctions, distinction between acts of decision and acts of data collection, collaborative and/or distributed actions, sources of evidence, etc, become essential to clarify in order to formalize the knowledge correctly and create a design for interactive clinical reasoning. An analysis of the qualities of the medical domain knowledge expressed in clinical guidelines is needed as well as a thorough formative activity analysis of how the actual clinical work is performed and could be developed. By taking an activity-oriented stand, i.e., keeping the activity as the focus of analysis (with an early DMSS prototype integrated), an activity model was created for the purpose of serving as a tool for:

- transforming informal structures and knowledge into formal knowledge in a CDSS,

- identifying ambiguities and inconsistencies in domain knowledge and clinical practice, and integrating means to handle these in the interaction with the CDSS,

- distinguishing between formalizable knowledge and knowledge that is better integrated through the design of interactive clinical reasoning,

- identifying different types of processes in order to provide support for respective and their interactions,

- identifying local organizational factors posing constraints on the design and use of a CDSS, and distinguishing general/universal and transferable knowledge,

- future evaluations of the CDSS in different local clinical practices.

A secondary purpose of the study was to investigate to what extent activity theory, which commonly is described and applied in an informal and general manner, can be used as base for knowledge acquisition and formalization work [2, $3,4,5,6]$.

The results presented in this paper of the case study, including the development and application of the activity model, are used as a bench-mark for further development of the theoretical foundations for knowledge structures to be implemented in the system, for the design of interactive clinical reasoning, and as a base-line for evaluation studies in clinical practice. 


\section{Methods, Materials and Procedure}

A prototype of the system DMSS is developed, which contains support mainly for assessment of evidence in a clinical diagnostic reasoning process aimed at supporting physicians in their main tasks. In our case study the prototype was integrated as a prompting tool for discussions about content and design of the system. However, the main focus was the investigation process as a whole.

Five patient cases investigated for a suspected dementia disease were in focus for this work. The studies were conducted in the setting of an out-patient ward at a geriatric clinic which investigates patient cases with suspected cognitive diseases. The patients were selected by including all new cases who were willing to participate in the study and who was assigned a particular physician from a certain date. We use the term investigation to denote the sum of diagnostic actions, interventions and follow-up actions, executed at this particular clinic. The time period of investigation for each patient was in the range of 3-10 months.

The investigation process was documented in the electronic health record (EHR), and by observations of clinical encounters and interviews. An approach inspired by grounded theory was used as method for analysis in order to identify general purposes and structures of activity, however, combined with the theoretical framework of the cultural-historical activity theory for integrating an understanding of the characteristics of particular tools, conflict situations and reasons for changes in purposes and structures $[2,3,5]$. The semi-formal structure that emerged, was applied at later stages of analysis to patient data in order to investigate to what extent it fits the data, and the structure was modified as a result. With the activity model as base, a process of differentiation of knowledge is ongoing, with an emerging knowledge base and a design of UI as result. The model is used as a dynamic reflective conceptual artifact in terms of [7] in the process of developing the system, which can be understood both by medical personnel and system developers, thus, having the potential of bridging the problem of transferring knowledge between the different communities.

\section{Results}

The results are organized into three sections. The activity model, which frames the clinical investigation activity in focus for our case study, is described in Section 3.1. Knowledge-based structures evolved based on the activity model are introduced in Section 3.2. Implications for and a proposed design of the DMSS user interface (UI) are presented in Section 3.3. 


\subsection{Activity Model}

We take as starting point, the view that the clinical activity of investigation essentially consists of two parts; the clinical process with its actions and subprocesses, and an activity system in terms of activity theory, which includes the object for activity, resources involved and their relations, and which imposes constraints on the execution of the investigation activity. The distinction is made for ontology and knowledge representation reasons, enabling a distinction between formal and informal described routines, treatment protocols and work divisions in the organization from what actually is implemented and applied in the execution of activity in a certain situation. A necessary property of the anticipated model is an ability to capture dynamic and changing situations and objects. We use the notion of object for data and phenomenon such as symptoms or diseases, when referring to what is in focus for each action.

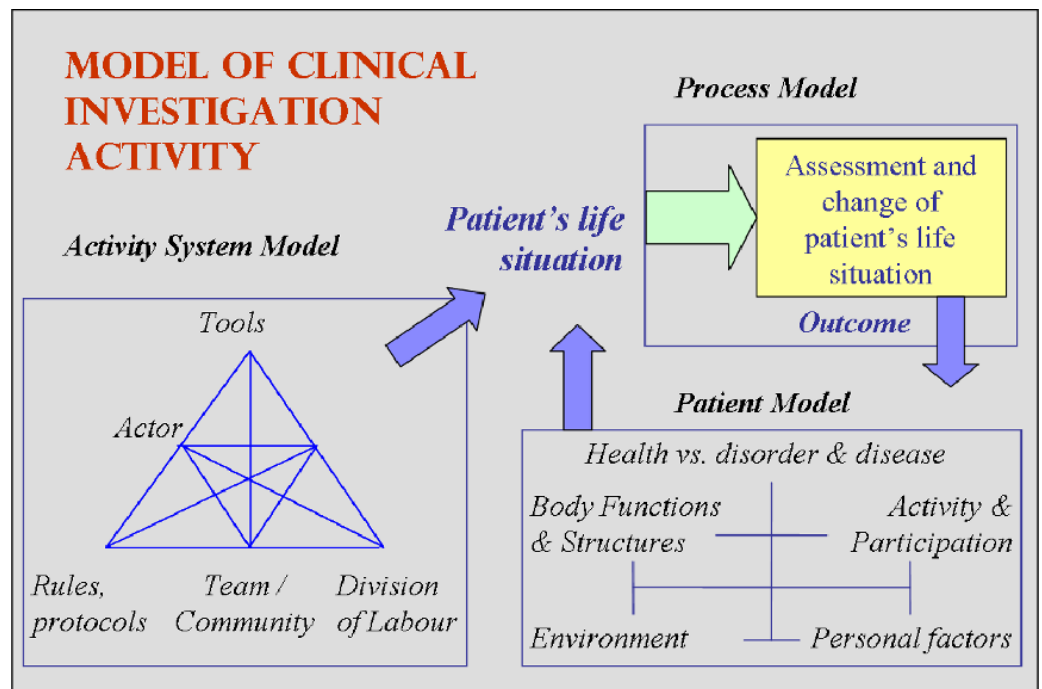

Figure 1. Activity model consisting of an activity system model based on [3], a patient model based primarily on [8], and a process model integrating the former two in the execution of an activity.

The model is based on cultural-historical activity theory (CHAT), which is a suitable framework for our purposes in that it integrates both systemic components such as resources and constraints, and emphasizes change and development of purposeful activities [3, 5]. The theory describes human activity as a dynamic system restricted by a set of conditions and inter-relations between the parts of the activity system and between systems. The theory defines an activity by the motive of the activity, driven by conscious or unconscious needs of a subject, or actor. The anticipated outcome of the whole clinical investigation process is identified as 
an optimal and satisfactory life situation for the patient, which can also be seen as the motive for and definition of this particular activity, and that guides the execution of the activity. It is important to take the patient-oriented view of the activity, since although it is the same type of activity in the investigation of two different patients, the execution will most likely differ, due to different needs of the patients, available resources and decisions made during the process. Therefore, we supplement the two analytic perspectives activity system model and process model with an elaborated object model, which in this domain is a model of the patient's life situation (Patient Model in Figure 1).

An overview of the activity model is provided in the following subsections, while a semi-formal description of components in the model and examples from patient cases can be found in [1].

\subsubsection{Activity System Model and Patient Model}

In terms of activity theory the activity system in focus includes the basic and original entities the subject, or actor, the object (here patient's life situation ${ }^{I}$ ) and tools, which are all entities with certain characteristic properties and roles in the activity system [5]. Their relations can be summarised by viewing the actor changing the patient's life situation (object and focus for activity) by using tools. The tools mediate the activity and should not be in focus for the activity.

For identifying the actor and the organisation he or she is representing, the activity system model viewed in Figure 1 can be used by defining the object as the main commission of the organisation [4]. For representation purposes it is convenient to distinguish the entities of an organisation, or actor in a wide interpretation, separated from the entities representing the patient's life situation (object). The patient model that is formed in the activity analysis presented in this work addresses terminology and ontological issues, issues of evidence identification, valuation, refinement, ambiguities, levels of granularity treated by different actors or professionals, the question of how to present the content to a user of a CDSS, etc. Therefore, in order to create a patient model that can function as a basis and instrument for formalisation of knowledge and outcomes of care activities, the patient model is based on nationally and internationally established classifications and terminologies created to structure knowledge concerning patients for different purposes. However, since the purposes differ, the resulting patient model is a synthesis of the WHO classification of functioning, ability and health (ICF) as base [8], supplemented with more elaborate and specific classifications of diseases and behavioural functions. Key components in the patient model are mechanisms for valuing the existence and amount of features,

\footnotetext{
${ }^{1}$ To be distinguished from the patient as an actor, participating in modifying his or her life situation.
} 
and their change over time, for decision support purposes, for evaluating care outcomes and for medical research purposes.

\subsubsection{Process Model and Typing of Actions}

The activity system involved in the execution of a task can be defined for each sub-action in the investigation process, as well as for the whole activity. The outcome of each action is another piece of evidence, related to the patient. Consequently, typically each action concerns a change of the incomplete knowledge about the patient's situation, and capturing this change is crucial in the design process.

When the investigation process as performed in the case studies was analysed, different sorts of actions were distinguished by the purpose, or the goal of a particular action. Typically, the actions were aimed at either collecting raw data $(\mathrm{CO})$; refine or interpret raw data or knowledge (RO); using the interpreted data for higher level of reasoning for the purpose of determine the existence of a phenomenon (DEO); determine the type of a phenomenon (i.e. increase level of granularity in the knowledge) (DTO); impose changes to the phenomenon in focus $(\mathrm{CHO})$ and value the effects thereof (ECHO). The actions of higher complexity which aim at a holistic assessment and change of a phenomenon we denote investigation actions (IO), which typically integrate all the other purposes. In addition, actions were identified which aim at controlling and directing the process (DA). Examples of such actions are referrals for examinations, involving colleagues for consultations, assignment of team-members to tasks and teammeetings. There are also actions, which aim at providing the patient, relatives and home care with information. These actions are interventions, aimed at changing the knowledge in the patient and/or the relatives or home care personnel (actions of type $\mathrm{CHO}$ ). Actions of the type $\mathrm{CHO}$ and ECHO typically belong to the intervention-part of the investigation activity.

Some of the sub-processes involved in the activity are viewed in Figure 2. The levels distinguished by lines in the figure indicate the different levels of complexity in the actions. As can be seen in the figure, the activities of analytic and decisive character are organised in the upper levels. The actions defined at these levels are typically defined as necessary for the main activity to be executed, according to the clinical guidelines and the domain knowledge. However, the level of necessity can differ depending on which guideline is used as tool for reasoning.

The object-creating actions, which are typically automated processes (i.e. operations) for the experienced actor, are organised at the bottom of the structure. Automated in the sense that the experienced actor does not have to put mental load or conscious thought on the execution, instead the actor can focus on the larger perspective and motive for the activity of which the action constitutes a part. These actions are typically administration of tools such as execute tests and examinations involving the patient. The actions defined at the lower levels in our 
analysis can very well be exchanged by other actions, which produce equivalent information necessary for accomplishing the decisive actions. Typically, this is the case when the routines and priorities at different workplaces direct the usage of different tools in the process, for instance, which radiology methods to use. Differences in which tools that are used may also due to different habits and values hold by individual actors.

The participation of patient and/or patient's family is necessary in the objectcreating actions at the lowest level, however, not at the other levels. This gives suggestions on how a decision-support system should be designed and for what kind of clinical situations, depending on which kind of actions are to be supported. There may be less need of a support system in the clinical patient encounter situation for the experienced actor when the examinations for obtaining evidence are executed. However, at this level different clinically validated methods as well as ad-hoc methods can be used. The less experienced actor can be supported in the choice of methods and in the execution of these sub-actions as well as in the higher-level actions of diagnostic reasoning. 


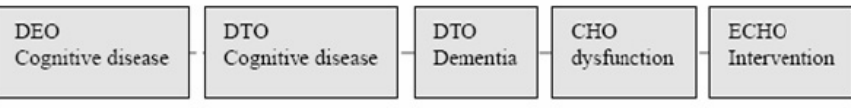

Level 3. Main reasoning process

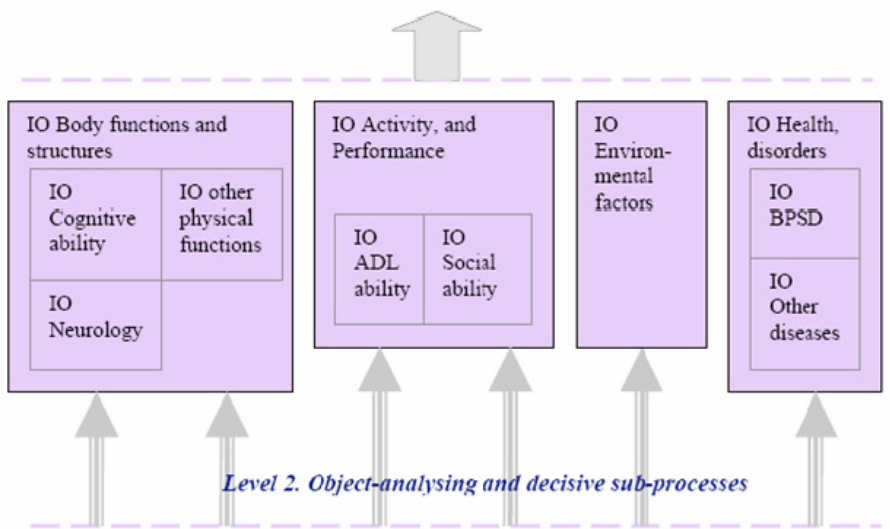

Level 1. Object-creating sub-actions

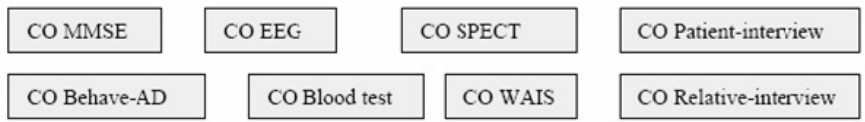

Figure 2. Different levels of complexity in the process of investigating cognitive diseases. BPSD is an abbreviation for Behavioral and psychological symptoms in dementia, and MMSE, EEG, WAIS, SPECT and Behave-AD are abbreviations for clinical examinations.

By distinguishing actions by their purposes a model is created, which identifies reasoning processes and logistic processes and their respective sub-actions, including their characteristics (e.g., complexity, dependencies, suitability for formalization, required resources and constraints for completion, etc.). Based on the model formal modules for decision support are created and supplemented with functionality provided through the interaction with a system.

\subsubsection{Levels of Complexity}

In our case study, three main dimensions of the process were distinguished, representing different levels of complexity. For practical reasons the two perspectives were distinguished from a logistic (or administrative) perspective, considering that the CDSS is primarily aimed at supporting higher-level reasoning. The logistic process became visible in the event-based documentation in the electronic health record (EHR) and in the scheduling of the patient's 
encounters with different personnel and for examinations. However, the quality of the information included in the EHR is typically raw data, which needs to be refined to be useful for reasoning, or summaries of coarse granularity, also not particularly useful for the level of reasoning that is needed for diagnosis. Therefore, the actions of higher complexity leading towards qualified decisions are focussed in our analysis, where raw data is being refined and interpreted within different frames of reference. Such actions are partly supported in the prototype system by production rules, based on clinical guidelines in the domain and formulated in knowledge-building sessions with domain experts. The basic actions of collecting raw data (CO) are supported by the system in that suggestions are given of which methods or clinical instruments to use, of which some are integrated in the system as basic data collecting frames. The logistic perspective was in the case study taken into consideration when conflicts between the perspectives occurred. Typically, conflicts were caused when the reasoning process had to be adjusted to the routines, time-scheduling constraints and bottlenecks in the organisation.

Two levels of reasoning processes from a physician's perspective can be identified in the activity. The main reasoning includes diagnostic reasoning and decision-making concerning interventions. Supportive reasoning processes involve investigations of specific domains in an individual, such as neurology, cognition, psychology, physiology, etc. (Figure 2). These domains correlate to the categorisation made in the patient model (Figure 1). The different purposes of these processes can be summarised by viewing the supportive processes as investigating dysfunction per se, while the main reasoning relates the dysfunctions in the perspective of a possible dementing disease. The three views of the process (logistic process, investigation of specific domains and the main reasoning process) are executed in parallel, they are cross-fertilizing and dependent on each other, and are partly overlapping depending on circumstances in the environment.

A similar structure is seen if viewing the contributions to the investigation process from other professional's perspective. The granularity of evidence differ between the different professional categories when they investigate the different domains of the patient model, which generates a richer synthesis through the different professional contributions than when an investigation is done by the physician alone, as is the case in some care environments. Therefore, the patient model needs to be elaborate enough to also capture evidence from other professional perspectives than the physician's, and consequently, the UI should integrate means to support all professional's work and reasoning processes that are involved in a patient case. By using ICF as basis for framing the patient's situation, different fields of expertise is covered at Level 2 (Figure 2). 


\subsection{Knowledge-Based Structures in DMSS}

The model includes an analysis of the international domain knowledge based on evidence-based medicine (EBM), which represents a category of tools in the activity system model (Figures 1,3). The knowledge is interpreted in clinical guidelines, treatment protocols, however, informally described, often ambiguous and incomplete [1,9]. For knowledge representation purposes a distinction is made based on the activity model of which knowledge is suitable for formalization in a rule-based knowledge base and which knowledge needs to be mediated through the design of the UI. A goal of the design of the system is to present the domain knowledge including its limitations. This is done by providing the user the evidence in a patient's case visualized and interpreted within different and sometimes conflicting frames of interpretations. These frames represent clinical and internationally validated tools for categorization, terminology, and screening. The frames can also refer to different clinical domains of expertise. In order to support the reasoning process, this functionality also provides the user support for creating bases for, and valuating diagnostic hypotheses at different point in the process. This is accomplished by integrating a meta-level in the knowledge base, which handles the ambiguities, the process components, the local and individual preferences and, consequently, the interaction with the user. The meta-level knowledge structure uses formalized knowledge from different clinical guidelines, synthesizes and ranks these sources in interaction with the user, while leaving the final decisions to the user when the evidence in a patient case is ambiguous.

As a result of the activity analysis, a strong orientation to support for processes is included in current design process, since there are supplementary professions working towards the goal of achieving an optimal situation for a patient, each with their reasoning processes and frames of interpretations. A key issue is the structure, content and visualization of the patient model, which should function as a common tool for communication and cooperative work. Furthermore, the patient model also serves as a basis for data structures to be implemented in databases, serving purposes of follow-up of individual patients as well as evaluation of care provided by an organization. In a longer perspective, the information needs to be useful for evidence-based medical research, which puts additional quality requirements on the data structure in order to obtain sustainability.

The basic rulebase (based on international evidence-based knowledge and guidelines to the extent they can be formalized in a rigorous way), the metarulebase (containing and handling additional knowledge concerning differential diagnosis, ambiguities, local preferences) and the data structure based on the patient model (structured according to international classifications) need to be formalized using different formal techniques in order to fulfill their purposes. The activity analysis reveals at a semi-formal level their respective requirements and which knowledge items to be handled by the different knowledge structures. Ongoing research addresses the possibility to integrate these into a common 
formal framework based on general logics in a fundamental view of transformations between different logics $[10,11,12,13]$.

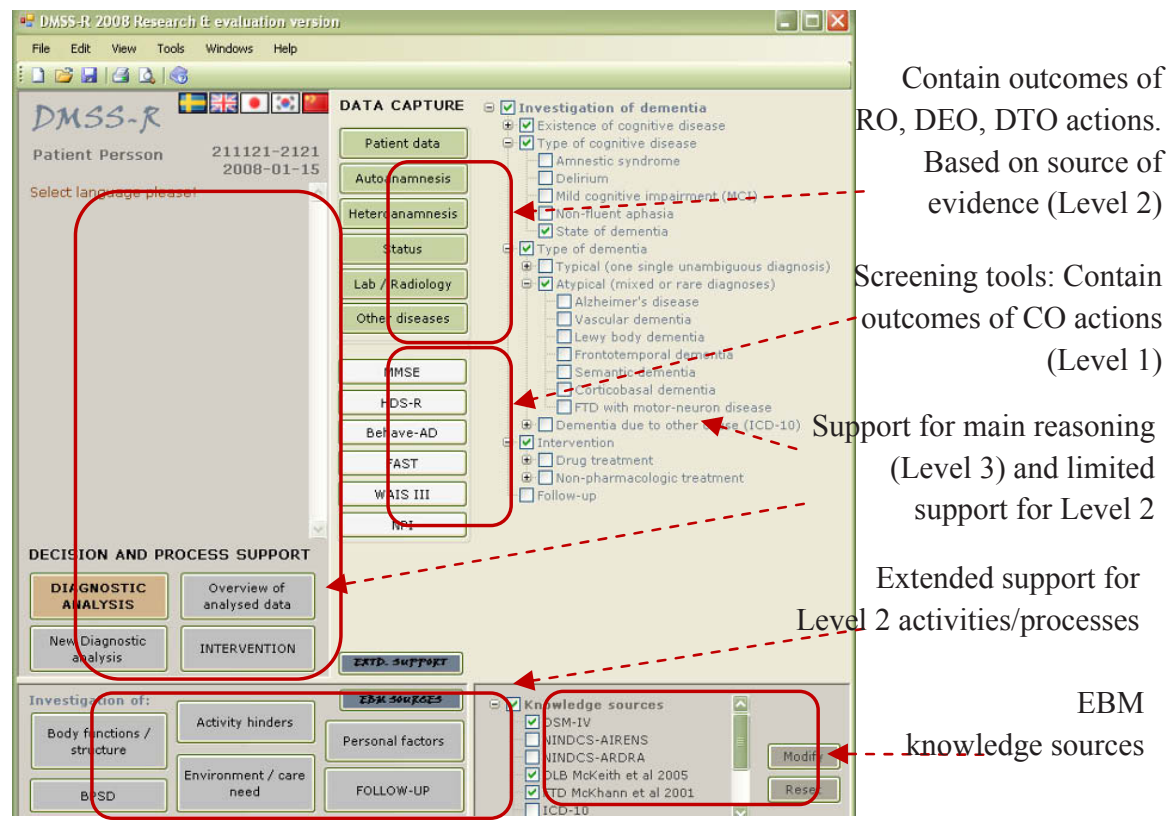

Figure 3. Design of the main frame of DMSS.

\subsection{Activity-Oriented Interaction Design}

We argue that the basic components of the activity model, i.e., process, tools, rules and regulations, cooperative aspects, patient model, etc., need to be reflected in the UI of a CDSS. The design of the DMSS UI is tightly intertwined with the meta-level of the knowledge base, which directs the use of the system and provides the support that is needed at different points in the process. This part of the system needs to be flexible, so that local and individual preferences can be met, such as which clinical guidelines and screening tools to use. In Figure 3 an initial re-design of the system is shown, in which the different categories of components correspond to different levels of activity in the investigation process. A major distinction is done between containers, or tools, for data capture and components for analysis and decision support. Further distinctions and categorizations of data capture functionality are made based on sources of evidence and validated screening tools, in order to obtain reliable data. The advanced support for reasoning is based on the main reasoning process (Level 3 in Figure 2) with a limited support for supportive reasoning processes (Level 2 in 
Figure 2). This functionality is currently being supplemented with extended support modules for these processes, developed from a teamwork perspective.

\section{Conclusions and Future Work}

The presented work provides an activity-theory based conceptual model for a clinical investigation activity. The model is based on analyses of the domain knowledge and case studies of investigations of actual patients and is used to frame the investigation process, as an alternative method to the formal ontology and workflow approaches. One major purpose is to identify relevant actions to formalize for decision support in the system and actions to support by the design of interactive clinical reasoning. The model can be viewed as a bridge between the informal view on clinical actions obtained in field studies and the formal implementation of the reasoning using suitable formalisms for representation.

Activity theory has previously been crystallized into tools for e.g., system development and evaluation [2, 4, 6], for methods for collaborative knowledge building [7] and development work research [3]. This work takes the use of the theory a step further, by integrating the theory in a semi-formal framework for clinical knowledge-based system development. A theory-based assessment model for clinical activity is presented, which is currently being used, developed and evaluated in the process of developing a decision-support system for dementia care. The approach is activity-oriented and integrates support for processes as well as the creation of a shared understanding of, and a common ground for activity, i.e., the patient's health and well-being. The model frames the knowledge to be integrated in the system, in what way different knowledge structures is best mediated to the user and in what way the system can be used as a tool for development of knowledge and skills in an individual user or in a team, as well as develop collaboration in health care.

Furthermore, the need for methods that can bridge the gap between system developers and medical professionals in the development of clinical knowledgebased systems is addressed. The activity model (partly crystallized into prototypes in the process) constitutes a product of a collaborative knowledge building process, both for different health care professionals at different levels of care, but also for persons with different professional backgrounds in the development team.

Since the work presented in this paper is based on a limited amount of patients and focusing a specific domain, the activity model and implementations based on the model will be further evaluated and developed using additional patient cases. Furthermore, the framework will be used for developing knowledge systems in different domains. Ongoing work includes evaluations of DMSS in clinical settings concerning interactive support for hypothesis building, as well as evaluation of the basic knowledge base. The theoretical development of a 
framework for knowledge-based structures is continuing, in addition to the implementation of the patient model and extended support for teamwork.

Acknowledgments: We thank patients and physicians who have participated in evaluations. The DMSS-project is partly funded by Swedish Brain Power.

\section{References}

1. Lindgren, H.: Decision Support in Dementia Care - Developing Systems for Interactive Reasoning. (2007) http://urn.kb.se/resolve?urn=urn:nbn:se:umu:diva-1138. Accessed 4 Feb 2008.

2. Bødker, S.: A human activity approach to user interfaces. Human-Computer Interaction, Vol. 4, pp. 171-195 (1989).

3. Engeström, Y.: Learning by Expanding: An Activity-Theoretical Approach to Developmental Research. Helsinki, Orienta-Konsultit (1987).

4. Kaptelinin, V., Nardi, B., Macaulay, C.: The Activity Checklist: A tool for representing the "Space" of context. Interactions, July - August (1999).

5. Vygotsky, L.: Mind in Society: The Development of Higher Psychological Processes. Cambridge, Harvard University Press (1978).

6. Häkkinen, H., Korpela, M.: A participatory assessment of IS integration needs in maternity clinics using activity theory. Int. J. Med. Inf. Vol. 76, pp. 843-849 (2007).

7. Singh, G.: Investigating the support of reflective activities by collaborative technologies: an activity theory based research model. In: Proc. TT211C2006, pp. 49-58 (2006).

8. WHO.: International classification of functioning, ability and health: ICF. http://www3.who.int/icf/icftemplate.cfm. Accessed 4 Feb 2008.

9. Lindgren, H., Eklund, P.: Differential diagnosis of dementia in an argumentation framework. Journal of Intelligent \& Fuzzy Systems Vol. 16, pp. 1-8 (2005).

10. Goguen, J.A., Burstall, R.: Institutions: Abstract model theory for specification and programming. Journal of the Association for Computing Machinery Vol. 39, pp. 95-146 (1992).

11. Meseguer, J.: General logics. In: Logic Colloquium '87, pp 275-329 Elsevier, North-Holland (1989).

12. Eklund, P., Helgesson, R., Lindgren, H.: Towards refining clinical evidence using general logics. To appear in: Proc. ICAIS (2008).

13. Eklund, P., Lindgren, H.: Towards dementia diagnosis logic. In: 11th Int. Conf. Information Processing and Management of Uncertainty in Knowledge-based Systems (IPMU'06), 12512257. Editions EDK, Paris (2006). 